\title{
The Genetic Revolution: The Challenges of Genetic Sequencing in Substance-Use Disorders
}

\author{
Xochitl Longstaff* \\ Department of Bioengineering, Stanford University, USA \\ *Corresponding author: Xochitl Longstaff, Department of Bioengineering, Stanford University, 443 Via Ortega, Rm119, Stanford, \\ CA, 94305, USA. \\ To Cite This Article: Xochitl Longstaff. The Genetic Revolution: The Challenges of Genetic Sequencing in Substance-Use Disorders. Am J Biomed \\ Sci \& Res. 2019 - 3(3). AJBSR.MS.ID.000673. DOI: 10.34297/AJBSR.2019.03.000673
}

Received: May 17, 2019 | Published: June 11, 2019

Keywords: Next-generation sequencing (NGS); Genome-Wide Association Studies (GWAS); substance-use disorders; Addiction; Genes; Prevention; Professional Care

Abbrevations: NGS: Next-Generation Sequencing; GWAS: Genome-Wide Association Studies; SNPs: Single Nucleotide Polymorphisms

\section{Introduction}

Substance Use Disorders present a global public-health issue and are responsible for approximately $12.4 \%$ of deaths worldwide as well as injury or illness in over 40 million individuals every year [1]. Substance Use Disorders are associated with loss of productivity, higher levels of domestic violence, and increased propensity for criminal behavior and incarceration [2]. Due to the high numbers of people affected by this disorder and the large burden it places on society as a whole, developing effective treatment protocols to combat substance Use Disorders is necessary. The brain-disease model can reduce the stigma around addiction as it suggests that the likelihood of substance abuse is influenced by factors such as genetics and neural pathways rather than any moral shortcomings or poor decision-making skills [2]. Next-generation sequencing (NGS) and Genome-Wide Association Studies (GWAS) allow scientists to investigate genes associated with substance-use on a much deeper level [3].

\section{Genes and Substance Abuse}

Current models for Genetic Architecture of Human Dependence The general consensus for the genetic basis of human dependence on drugs is that it is affected approximately $50 \%$ by polygenic influences which each contribute a small amount to the likelihood of abuse [1]. The first major GWAS study for alcohol abuse was published in 2008 and analyzed genetic samples from 1,024 German males compared to a control sample of 1,358 subjects from separate population-based studies (487 from PopGen, 488 from KORA-gen, and 383 from the Heinz Nixdorf Risk Factors, Evaluation of Coronary Calcium, and Lifestyle (RECALL) [3]. All patients were diagnosed with alcohol dependence by two independent psychiatrists using the DSM-IV criteria and were separated by sex, ethnicity, age at onset and recruitment procedures; results were compared with a study in rats [3]. Through this method, the researchers found two strong markers: rs7590720 and rs1344694 on chromosome region 2 q35 which were previously linked to alcohol dependence. The gene most associated with these Single Nucleotide Polymorphisms (SNPs) was PECR which is an enzyme involved in fatty acid metabolism [3]. This study provides promising data that this variant may be involved with alcohol-use disorders by affecting the metabolic pathway in alcohol consumption.

Several studies have suggested that the endogenous opioid system is involved in the neurobiological pathway of alcohol addiction [4]. The A118G polymorphism of the OPRM1 gene causes a functional difference in the opioid receptors which cause the receptor to bind endorphin three times more strongly than other variants. One study showed that individuals with the $G$ allele had stronger feelings of intoxication, sedation, and happiness across all trials compared to the control, and were three times more likely to report a history of familial alcohol abuse [4].

Studies that examine the genes involved in the nicotine pathway and addiction have revealed several markers in the chromosome 15 gene cluster which encode for nicotine acetylcholine receptors such as the 3,5, and 4receptors and differ significantly between heavy and light smokers [5]. Identifying these types of genetic markers 
could help further understanding of the mechanisms involved with substance use as well as inform the way patients think about their substance use. Researchers have already begun to investigate the emotional impact of receiving genetic predisposition information for nicotine addicts. One such study surveyed 269 British adult smokers-both men and women with a mean age of 41.5 yearswho smoked at least 1 cigarette a day. The study investigated the individual's perceived control over stopping smoking as well as preferred cessation methods given their genetic results (i.e. "using my own willpower," "taking Zyban, a drug that reduces cravings for nicotine by increasing dopamine levels," "using nicotine replacement therapy such as gum or patches," "asking my family doctor for advice" etc.). This study did suggest that learning about a genetic predisposition to nicotine may increase the desire for effective cessation methods, increasing the likelihood of reaching out to get help [5].

While most genetic studies of addiction focus on specific Substance Use Disorders and particular genetic pathways, one study attempted to find potential addiction variants by converging genome scanning data from multiple studies [6]. The study analyzed more than 104 markers that have previously been found to have a positive correlation with addiction based on previous linkage studies. They then used the criteria of ' $5 \mathrm{Mb}$ ' which follows that positive markers linked to substance abuse vulnerability in at least two different samples lie within $5 \mathrm{Mb}$ of each other and found that 34 of the 104 markers fell into $155-\mathrm{Mb}$ chromosome regions. They found that 6 different human chromosome areas contained positive markers for addiction [6]. Further studies based on this analysis should be done to understand the molecular genetic pathway of those identified chromosome regions.

\section{Conclusion}

NSG and GWAS studies represent powerful tools for investigating possible genetic factors involved in Substance Use Disorders. The research in this area is still in its early stages and more work is needed to identify genes linked to different types of Substance Use Disorders and to uncover the mechanisms by which these genes act. Improving the understanding of genes involved in Substance Use Disorders could allow for more precise and effective treatment options as well as shift perceptions around Substance Use Disorders. Providing robust and informative genetic counseling be an essential part of the future genetic research and precision medicine for people with Substance Use Disorders to ensure that patients understand the complexity, limitations, and significance of their results.

\section{References}

1. Goldman D, Oroszi Gabor, Ducci F (2005) The Genetics of Addictions: uncovering the genes. Nat Rev Genet 6(3): 521-532.

2. Livingston JD, Milne T, Fang ML, Amari E (2012) The effectiveness of interventions for reducing stigma related to substance use disorders: a systematic review. Addiction 107(1): 39-50.

3. Treutlein J, Cichon S, Ridinger M, Wodarz N, Soyka M, et al. (2009) Genome-wide Association Study of Alcohol Dependence. Arch Gen Psychiatry 66(7): 773-784.

4. Ray LA, Hutchison KE (2004) A Polymorphism of the $\mu$-Opioid Receptor Gene (OPRM1) and Sensitivity to the Effects of Alcohol in Humans. Alcohol Clin Exp Res 28(12): 1789-1795.

5. Wright AJ, Weinman J, Marteau TM (2003) The impact of learning of a genetic predisposition to nicotine dependence: an analogue study. Tob Control 12(2): 227-230.

6. Uhl G, Liu Q Naiman D (2002) Substance abuse vulnerability loci: converging genome scanning data. Trends Genet 18(8): 420-425. 